\title{
Customised enriched acoustic environment for sound therapy of tinnitus
}

\author{
Pedro Cobo* (1), María Cuesta (1), and Carlos de la Colina \\ Institute for Physical and Information Technologies (ITEFI), Spanish National Research Council (CSIC), Serrano 144, Madrid, P.O. \\ Box 28006, Spain
}

Received 6 April 2021, Accepted 20 July 2021

\begin{abstract}
Tinnitus is an auditory disorder very difficult to treat. Whereas up until now there is not a "cure" for tinnitus, the most extended treatment combines counselling with sound therapy. When this sound is a broadband noise in the audio frequency band, this protocol is named tinnitus retraining therapy. Even though broadband noise was proposed at the beginning as the stimulus for sound therapy, many other sounds have been subsequently proposed and used, including tones, noise bands, music, and nature sounds. Although any sound, low enough to avoid annoyance, discomfort or hearing damage, is better than silence for tinnitus treatment, it is not still clear the relationship of the success of the therapy with the properties of the sound stimuli. The aim of this article is to propose an optimal sound treatment that provides a precise and selective stimulation of the whole auditory system. The proposed sound stimulus, Enriched Acoustic Environment, consists of sequential tones or broadband noise matched to the HL curves of the patients. The acoustical characteristics of these stimuli are analyzed and their positive effects in the treatment of subjects with tinnitus are reported.
\end{abstract}

Keywords: Tinnitus, Hearing loss, Sound therapy, Enriched Acoustic Environment

\section{Introduction}

Tinnitus is the medical term for the auditory perception of sounds in the absence of sound sources internal or external to the body $[1,2]$. This phantom auditory perception presents mainly as a continuous and fluctuant tone, ringing, or hissing of high or low pitch, with loud or soft sensation level. Tinnitus is an uncomfortable auditory disorder affecting severely the quality of life of subjects [3], causing many audiological, cognitive, and neurological concerns including altered sleep patterns, attention deficits, annoyance, irritability, panic, stress, anxiety, and depression [4-6].

Epidemiological studies afford that approximately $10 \%$ of the adult population in Western industrialized countries have been eventually affected by tinnitus [7]. For many of these subjects (approximately 1-2\%), tinnitus is a handicap producing distress and deteriorating significantly their quality of life. Approximately 5 million of European people have suffered of moderate-to-severe tinnitus [8]. In Spain, $17 \%$ of consultations in specialised audiological clinics are due to tinnitus [9], whilst $1 \%$ of population are affected of severe tinnitus [10].

It has been suggested that hearing loss (HL), the rise of hearing thresholds of a subject expressed in decibel $(\mathrm{dB})$, is a significant risk factor for developing tinnitus. The occurrence of hearing problems in the developed countries has

*Corresponding author: pedro.cobo@csic.es increased over the past last years, due to aging of the population (age related hearing loss, ARHL) and overexposition to noise (noise related hearing loss, NRHL) [11]. The incidence of tinnitus, as ARHL does, increases with age. Loud noises, such as those from industry, traffic in urban environment, and portable music players in young population, are common sources of NRHL and increase the risk of suffering tinnitus. Exposure to noise during short time, such as attending a disco session, can produce tinnitus that usually goes away, but living with long-term harmful noise can produce permanent damage to auditory system and hence raises the risk of developing tinnitus [12]. For developed countries, this renders a higher incidence of tinnitus, which in turn has a significant economic impact on the public health system [13]. Although HL can be a risk factor for tinnitus, it may apparently occur in people with normal hearing thresholds. Recent studies have disclosed that high frequency HL (above $8 \mathrm{kHz}$ ), which is not currently measured in hearing screening, and hidden hearing loss (HHL), a type of permanent cochlear damage without an elevation of hearing thresholds, can afford tinnitus [14, 15].

Therefore, tinnitus is a quite prevalent disorder challenging to deal with. There is evidence that tinnitus is associated to adjustments of the normal operation of the neural part of the auditory system which attempts to compensate for some kind of deficit at the auditory periphery [16]. When there is some deafferentiation from the peripheral downstream to the neural auditory system (a loss of hearing, 
P. Cobo et al.: Acta Acustica 2021, 5, 34

for instance), the neural part reorganises to compensate for this deficit. This capacity of reorganization is a characteristic of brain plasticity. There exists physiological evidence of functional changes in the neural part as a reaction to damages in the peripheral part of the auditory system, such as these produced by an acoustical trauma [17], head trauma, ear infection, ototoxicity, vestibular troubles, and others, giving rise to tinnitus.

The deafferentiation of a normal input to central auditory system represents a potential trigger of plastic changes, such as increasing of the spontaneous activity (hyperactivity), hypersyncrony or tonotopic map reorganization [16]. These three mechanisms could underpin the origin of tinnitus at sub-cortical and/or cortical levels of the auditory system through the following sequence [18]: (1) a problem in the auditory periphery (for instance, hearing loss due to noise overexposition or aging) is produced; (2) deafferentiation in the damaged frequencies could afford an overrepresentation of the frequencies at the edge of the lesioned band, which causes hyperactivity and/or possible hypersynchrony in cortical pathways, giving rise to the primary tinnitus signal; (3) under normal functioning, the tinnitus signal is inhibited at the thalamic gate by a feedback loop (inhibitory gating mechanism). When this gating mechanism is compromised, inhibition fails, and the neural signal is transmitted to higher order structures, where it causes permanent reorganization and chronic tinnitus.

Symptomatic tinnitus can be temporarily masked by sound stimulation aimed to produce residual inhibition (RI) [19]. The tinnitus signal usually returns back some time after (from minutes to hours) the acoustic signal ceases. An acoustic signal which yields a long lasting RI after the stimulus is ceased should become an effective sound therapy. The dependence of the inhibition grade on the sound characteristics, such as frequency contents (peak, bandwidth), sound level, time length, and other parameters is still under study [19]. Many sound therapies for tinnitus treatment have been suggested [20-22] including tinnitus re-training (TRT) [23, 24], auditory discrimination (ADT) [25-29], phase-shift [30, 31], neuromonics [32], notchedmusic [33-35], neuromodulation [2, 36-38], fractal tones [38-40], and many others.

Sound therapies assume that exposing the auditory system to an enriched sound can rearrange it to normal functioning due to plasticity. Although any sound is better than silence for tinnitus treatment, provided that it does no annoy, create discomfort, or damage hearing [24], it is still not clear the relationship of the success of the therapy with the properties of the sound stimuli. The aim of this article is to propose an optimal sound treatment that provides a precise and selective stimulation of the whole auditory system. Since this sound therapy is applied combined with counselling, in the context of a variation of the TRT, the bases of this tinnitus treatment are reviewed in Section 2. The fundamentals and acoustic properties of the proposed therapy, named Enriched Acoustic Environment (EAE), are exposed in Section 3. Some results are presented in Section 4. And finally, the main conclusions of this article are outlined in Section 5.

\section{Tinnitus retraining treatment (TRT)}

Tinnitus is a hearing disorder that arises by aberrant plastic compensation mechanisms in the neural auditory system to some peripheral deafferentation. Thus, tinnitus is in fact a phantom auditory perception generated at subcortical or cortical levels of the auditory system. In spite of many efforts carried out to find a "cure" for tinnitus, including pharmacological drugs, sound, magnetic/electrical stimulation, physiotherapy, relaxation, and other complex interventions (defined as a combination or two or more of the preceding modalities) [41], such a cure there not exists nowadays. Rather than looking for removing it, the practical guideline for treating tinnitus is trying to reduce its distress.

The most extended treatment of tinnitus is TRT, a comprehensive, non-invasive protocol applied in most clinics that has demonstrated to achieve about $80 \%$ of distress reduction in tinnitus patients, based in the neurophysiological model of Jastreboff [23]. This model is based on that, whilst the auditory system is involved in the trigger and propagation of the tinnitus signal, other brain systems, mainly the limbic and the autonomic nervous systems, are involved in its deleterious reactions, beginning with annoyance, panic, and difficulties with sleep and concentration, and, in the worst cases, evolving into anxiety, stress, and depression.

Sound, an acoustic signal at the peripheral auditory system, is transduced into a sequence of spikes at the hair cells-auditory nerve interface. The sound evoked spikes propagate downstream the afferent auditory way from the auditory nerve (AN) to the auditory cortex (AC) through the cochlear nucleus $(\mathrm{CN})$, the olivary complex $(\mathrm{OC})$, the lateral lemniscus (LL), the inferior colliculus (IC), and the medial geniculate body (MGB) [11]. Sound processing also activates other brain systems, such as the amygdala and the hippocampus, two major limbic structures. The amygdala is particularly sensitive to voice and music and plays a significant role in conditioning to auditory fear and regulation of the response to sound startle [42]. A stressful acoustic stimulus, may release amygdala-mediated stress hormones via the HPA-axis, which may have negative effects on health. A short-term exposure to stress hormones may produce hearing protection [42]. Noise exposure can affect long term plasticity of hearing [42]. The hippocampus can add temporal dimension to the auditory processing.

Sound patterns originate subcortically and enter both auditory and limbic systems via the MGN. Under normal functioning, the limbic system may recognize a transient tinnitus as perceptually unsuitable and inhibit it at the MGN [43], this in turn reducing the propagation of the unwanted tinnitus signal in both circuits. When inhibition of this tinnitus signal is prevented, continued thalamocortical activity results and the constant perception of the tinnitus signal is produced (chronic tinnitus). Tinnitus, thus induced by hearing disorder, is associated to changes in the hippocampus. The dysregulation of limbic and auditory networks is in the basis of chronic tinnitus, indicating that an advanced comprehension of auditory-limbic interactions 
may be crucial for an ultimate cure of tinnitus in the future $[42,43]$.

The aim of TRT is to habituate to the tinnitus signal by reducing its negative response. When the tinnitus signal propagates to other brain systems, and in particular triggers the limbic and autonomic nervous systems, brings a number of aversive reactions and tinnitus becomes annoying. Consequently, the aim of treatment should be taking advantage of brain plasticity to achieve the habituation of negative tinnitus reactions, by blocking the functional connections that spread the tinnitus signal from the auditory to the other brain systems [23].

In practice, TRT combines counselling with sound therapy, both based on the above described neurophysiological model of tinnitus [23]. The main goals of sound therapy and counselling are to lessen the strength of neuronal activity related to tinnitus and to demystify it by unravelling the mechanisms underlying tinnitus, respectively [44]. Successful counselling will diminish the negative associations with tinnitus, which is necessary to facilitate its habituation. The sound stimulus is intended to maintain an enriched environment of non-annoying sounds that improve the treatment result by modifying auditory processing at subconscious levels and facilitating the habituation to the tinnitus perception [23].

An important aspect of TRT is that the aetiology of tinnitus is irrelevant, since it does not attempt to suppress the tinnitus signal itself but to attenuate its negative reactions. Any type of tinnitus can be successfully treated by TRT. As the tinnitus signal is prevented from reaching brain systems, the negative reactions significantly attenuate, being not necessary to remove them. This feature differentiates TRT from other methods more oriented to the suppression of the tinnitus source [23].

\section{Enriched Acoustic Environment (EAE)}

\subsection{Antecedents}

Noreña and Eggermont [17] demonstrated that the reorganization of the tonotopic map of cats produced by the exposition to an injuring noise could be inverted by exposing them to an appropriate EAE. Fourteen laboratory cats were exposed first to a loud noise environment. Seven of these cats (group 1) were not subjected to additional acoustic stimulation, whereas a second group of other seven cats (group 2) were immersed into an EAE inside a room. The EAE consisted of a high-pass filtered tone-pip sequence (see Sect. 3.2) of random frequency between $625 \mathrm{~Hz}$ and $20 \mathrm{kHz}$ and sound pressure level of $80 \mathrm{~dB}$. This level was above the expected HL in the high frequency range, but low enough to avoid further impairment to their hearing system. The cats of group 2 were stimulated for $24 \mathrm{~h}$ /day during 35 days to obtain the best effect on the auditory system after the traumatizing hearing loss. Other control group of 5 non-exposed cats was used. They mapped the auditory AI cortex of the three group cats. The tonotopic map of the traumatized non-EAE exposed cats (group 1) showed a profound reorganization compatible with high frequency hearing loss. However, the cortical map of the traumatized EAE exposed cats (group 2) showed a frequency organization similar to that of the cats in the control group. Therefore, they concluded that the cortical tonotopic map reorganization of cats produced by the traumatizing noise could be compensated by stimulating with a tone-pip EAE in the damaged frequency region.

Schaette and Kempter $[45,46]$ used a computational model of auditory neurons to demonstrate that tinnitusinduced hyperactivity produced by homeostatic plasticity could be reversed by appropriate acoustic stimulation. Using their model, they were able to calculate the firing rate and gain of downstream auditory neurons. The effects of homeostatic plasticity in these neurons were taken into account by introducing changes in the gain factor triggered by deviations of the main activity from a certain target activity (hyperactivity), so that the gain adjustments mimicked the changes in effective response by homeostatic scaling. As a result of this model, when the excitatory input to one of these neurons was reduced (deafferentation), homeostatic plasticity increased the effective gain resulting in tinnitus. They were also able to evaluate the effect of different sound stimuli (sound therapy) to these tinnitusdamaged neurons. When a white noise was used, none effect was observed in the aberrant gain produced by tinnitus. If, however, a matched-noise stimulation (a stimulus with spectrum matched to the HL curves) was applied to the damaged neuron, the aberrant gain was eventually removed.

Noreña and Chery-Croze [47] studied if the aberrant central gain distinctive of hyperacusis patients could be reduced by subjecting them to an EAE, consisting of a sequence of tone-burst. The auditory hypersensitivity of these patients could be caused by hearing losses, which produced a deficit of sensory inputs to the central auditory system, and hence plasticity-induced increase in central gain. The aim of the study was to test whether this aberrant central gain could be compensated by the use of an EAE and the auditory hypersensitivity consequently reduced. The EAE was composed of a sequence of tone-bursts of random frequency within the hearing range, and amplitude weighted as a function of the hearing loss. The participants were asked to hear the EAE for 1-3 h/day at a just audible level. After 15 weeks, all participants reported a statistically significant sensitivity decrease. Herráiz et al. [48] demonstrated that this therapeutic tone-burst EAE was able to produce clinically relevant relief in 21 tinnitus patients.

\subsection{Gammatone sequence}

Therefore, sequences of EAE have been used until now with either tone-pips or tone-bursts. Both consist of random frequency tones with amplitude weighted by a factor proportional to the HL and shaped by a window that is symmetrical, for the case of tone-burst, and asymmetrical for the case of tone-pips. The equation for such an EAE sequence is then, 

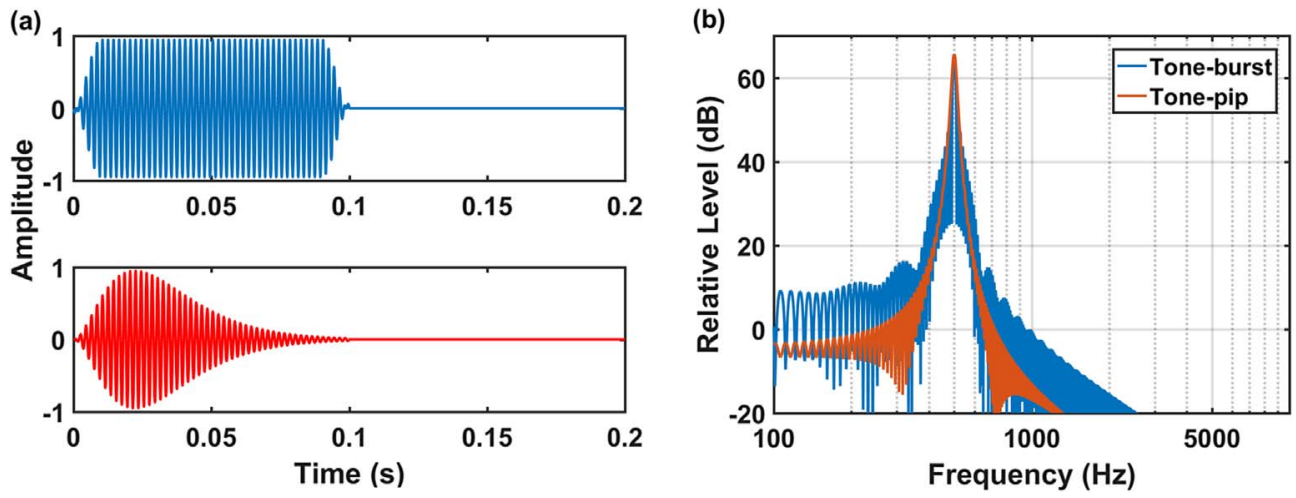

Figure 1. (a) A tone-burst and a tone-pip of frequency $500 \mathrm{sHz}$. The envelope of the tone-burst corresponds to a rectangular window of $0.1 \mathrm{~ms}$. The $(\alpha, \gamma)$ parameters of the tone-pip are $(90,3)$. (b) The corresponding log-spectra.

$$
\operatorname{eae}(t)=\sum_{n} A_{n}\left(f_{n}\right) W\left(t-\tau_{n}\right) \cos \left[2 \pi f_{n}\left(t-\tau_{n}\right)\right]
$$

where,

$$
A_{n}\left(f_{n}\right)=10^{\mathrm{HL}\left(f_{n}\right) / 20},
$$

is the amplitude of the tone,

$$
W(t)=\left\{\begin{array}{lr}
W_{T}(t) & \text { for tone }- \text { burst } \\
t^{\gamma-1} e^{-\alpha t} & \text { for tone }- \text { pips }
\end{array},\right.
$$

is the envelope function, $W_{T}$ is a time window, $(\alpha, \gamma)$ are parameters, $f_{n}$ is a frequency within the hearing range, $\operatorname{HL}\left(f_{n}\right)$ is the hearing loss at this frequency, and $\tau_{n}$ is the interlatency between tones (the inverse of the rate, or number of pulses per second).

The envelope of tone-bursts corresponds to a time window (Hanning, Hamming, Blackman-Harris, etc.). The rising and decaying parts of the tone-pip window are controlled by two parameters $(\alpha, \gamma)$, which can be chosen so that its magnitude spectrum matches the tuning curve of different parts of the auditory system. Figure 1a shows two tones, a tone-bust and a tone-pip, of the same frequency $(500 \mathrm{~Hz})$ and with similar duration $(0.1 \mathrm{~s})$. Figure $1 \mathrm{~b}$ shows the corresponding log-spectra. The main difference between both spectra is the presence of strong side-lobes in the case of the tone-burst. The spectrum of the tonepip is more "clean", and asymmetrical, similarly to the frequency response curves of different sites of the auditory system. Therefore, it seems that the tone-pips are more refined sounds for stimulating the human auditory system. In fact, these sequences of tone-pips have been used to stimulate laboratory animals with EAE of varying values of $(\alpha$, $\gamma$ ) and rate (20 per sec and 1 per sec for fast and slow rate, respectively) $[17,49,50]$.

The parameter $\alpha$ can be used to control the length of the tone-pip. Figure 2 shows four tone-pips of $\gamma=3, f_{0}=500$ $\mathrm{Hz}$, and different values of $\alpha$. It can be clearly seen that the larger $\alpha$ the shorter the length of the tone-pip. The appropriate values of $(\alpha, \gamma)$ to model the frequency response of different parts of the auditory system (basilar membrane, auditory neurons) depend on the specie. Korn studied a
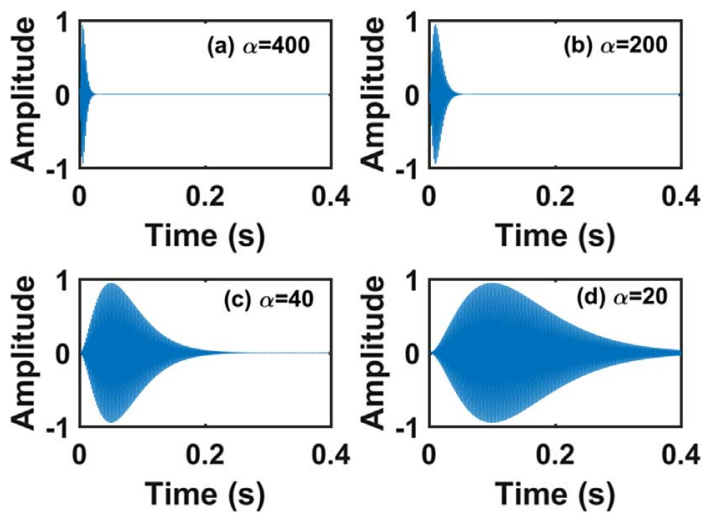

Figure 2. Some tone-pips for $\gamma=3, f_{0}=500 \mathrm{~Hz}$ and (a) $\alpha=400$, (b) $\alpha=200$, (c) $\alpha=40$, and (d) $\alpha=20$.

similar problem in the context of the theory of audio information [51]. According to the uncertainty principle, $\Delta f \Delta t=$ constant, any measurement of the frequency of a signal is affected by a zone of uncertainty, which is inversely proportional to the time (duration) of observation. The way our auditory system processes a signal is closely related with the uncertainty principle. A click is perceived as one of two consecutive sounds depending on its length. The mechanism allowing our auditory system to change from the time to the frequency domain in the processing of sounds is related with the concept of masking, which consists of the increment of the hearing threshold of a tone in the presence of another tone. The masking curve at each frequency is considered as the representative curve of this frequency. In other words, masking can be considered as a discretiser of the auditory system allowing to measure the discrete value of such frequency for a finite length stimulus. Therefore, the stimulus corresponding to the inverse Fourier transform of a masking curve can be considered as the elemental message at discrete frequency (EMDIF) [51]. When the stimulus is shorter than an EMDIF, its spectrum is wider than the corresponding masking curve and it is processed in the frequency domain. If, on the other hand, the stimulus is larger than an EMDIF, its spectrum is shorter than the masking curve, and it will be processed in the time 


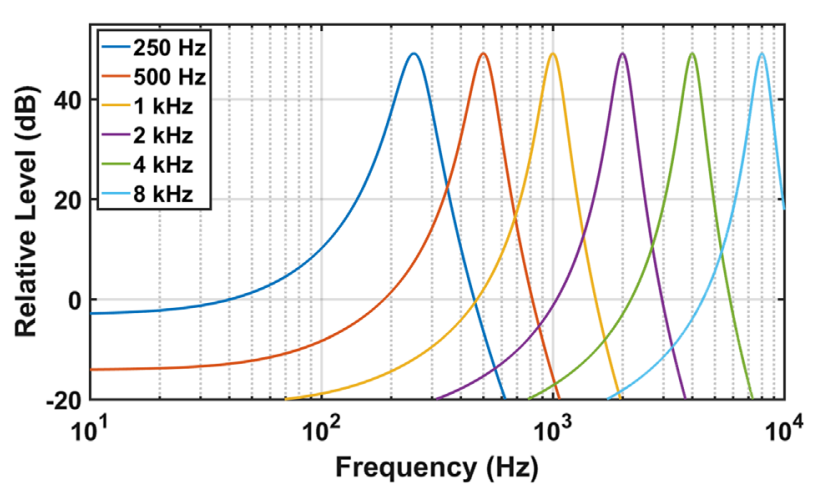

Figure 3. Normalised gamma filters for octave band frequencies between $250 \mathrm{~Hz}$ and $8 \mathrm{kHz}$.
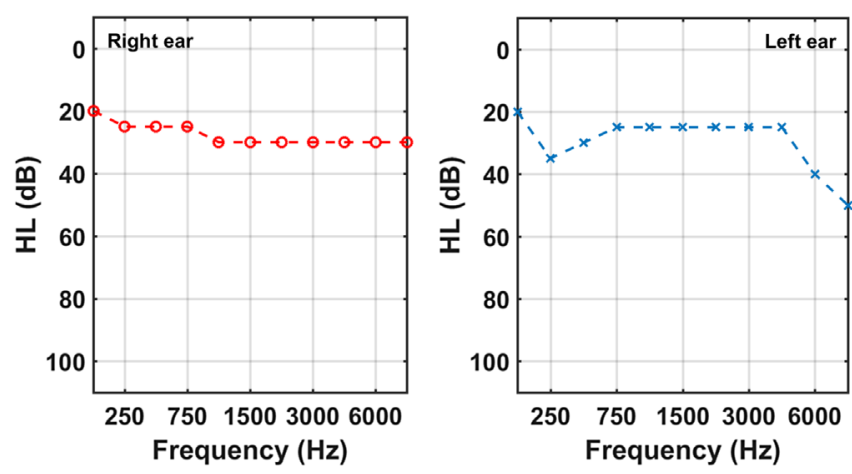

Figure 4. Right and left audiograms of a subject.

domain. Korn [51] showed that masking curves can be modelled by the filter,

$$
|S(\omega)|=\frac{\left[\alpha^{2}+\left(\omega-\omega_{0}^{2}\right)\right]^{\gamma}}{\omega^{\gamma-1}},
$$

which inverse Fourier transform is,

$$
e(t)=t^{\gamma-1} e^{-\alpha t} \cos \omega_{0} t,
$$

which corresponds exactly to a tone-pip.

An appropriate therapy for tinnitus subjects should be that with the two parameters $(\alpha, \gamma)$ tuned to the curves at various locations of the human auditory system. These are, in fact, the gamma filters [52-55]. The inverse Fourier transform of the gamma filters are the gammatones. Let,

$$
\mathrm{ERB}=0.108 f_{0}+24.7,
$$

be the equivalent rectangular bandwidth of an auditory filter, also known as critical band, where $f_{0}$ is the central frequency of the band. The corresponding gammatone is then given by Equation (5) with the pair of parameters $(\alpha, \gamma)$,

$$
\begin{gathered}
\alpha=2 \pi \mathrm{ERB}=2 \pi\left(0.108 f_{0}+24.7\right) . \\
\gamma=4
\end{gathered} .
$$

Therefore, just a parameter, the central frequency of the band $f_{0}$, determines the gamma filter at each frequency.
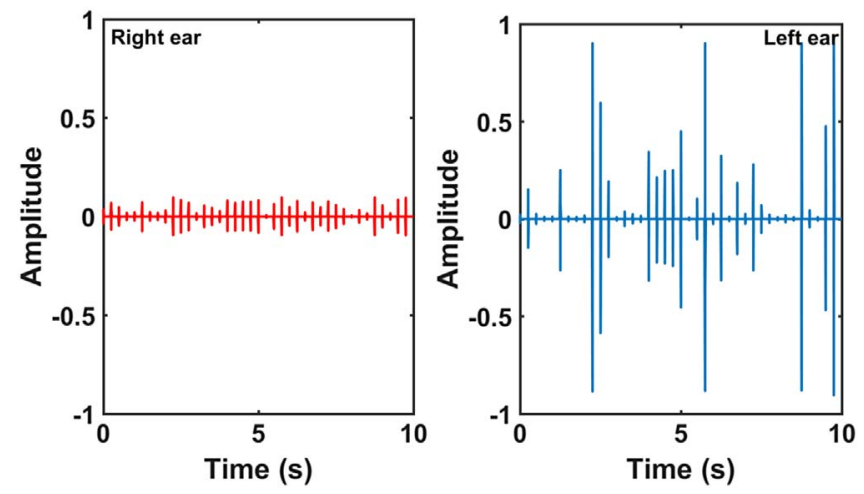

Figure 5. $10 \mathrm{~s}$ of a tone-pip EAE corresponding to the HL curves of Figure 4.

A bank of six normalised gamma filters at octave band frequencies between $250 \mathrm{~Hz}$ and $8 \mathrm{kHz}$, similar to the filters currently used for simulating the basilar membrane movement in the cochlea, is shown in Figure 3. Thus, a sequence of gammatones should be given by,

$$
\begin{aligned}
\operatorname{eae}(t)= & \sum_{m} A_{m} \frac{(2 \pi \mathrm{ERB})^{4}}{6}\left(t-\tau_{m}\right)^{3} e^{-2 \pi\left(0.108 f_{m}+24.7\right)\left(t-\tau_{m}\right)} \\
& \times \cos \left[2 \pi f_{m}\left(t-\tau_{m}\right)\right]
\end{aligned}
$$

where $A_{m}$ are the amplitudes, proportional to the HL values (Eq. (2)), and $(2 \pi \mathrm{ERB})^{4} / 6$ is a normalization factor [56].

Let us illustrate the design process of an EAE by gammatones for a subject with the pure tone audiogram shown in Figure 4. The subject exhibits mild losses (30 dB maximum) in the right ear and mild to moderate losses (minimum $20 \mathrm{~dB}$ at $125 \mathrm{~Hz}$ and maximum $50 \mathrm{~dB}$ at $8 \mathrm{kHz}$ ) in the left ear. Figure 5 shows the 40 gammatones of the first $10 \mathrm{~s}$ of each ear (10 s, 4 pulses/s). The maximum amplitude of the gammatones is normalised to 0.95 to avoid clipping effect. The amplitudes of the left ear are larger, as the HLs for this ear are higher. For each ear, the gammatones have amplitude proportional to the HL at each frequency and random frequency. The varying amplitude nature of the gammatones is best appreciated in Figure 6, where the spectra of the first 240 gammatones $(60 \mathrm{~s}, 4$ pulses/s) are shown superimposed to the left and right HL curves. Notice that there are gammatones at frequencies other than these measured by the audiogram. In fact, $\operatorname{HL}\left(f_{m}\right)$ is a function measured at some discrete frequencies (for instance $[125,250,500,750,1000,1500,2000,3000$ 4000, 6000, $8000] \mathrm{Hz}$ ). Thus, this discrete function must be interpolated on a constant frequency bin axis. The contour maps of the short-time Fourier transforms of the first 8 gammatones $(2 \mathrm{~s}, 4$ pulses/s) of each ear (Fig. 7) illustrate their random frequency characteristic. Notice that, whereas the gammatones have random nature along time, the left and right ears are stimulated at the same frequencies for each instant (diotic listening).

Hence, the sequence with gammatones provides an EAE customised for each patient, as it matches their HL. Furthermore, it affords the most selective and advanced 

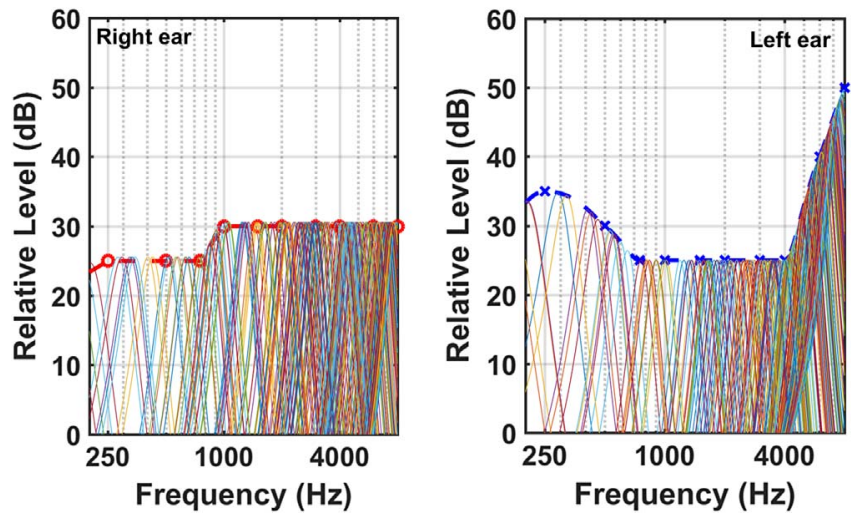

Figure 6. Log-spectra of the right and left tone-pips sequences superimposed to the HL curves.
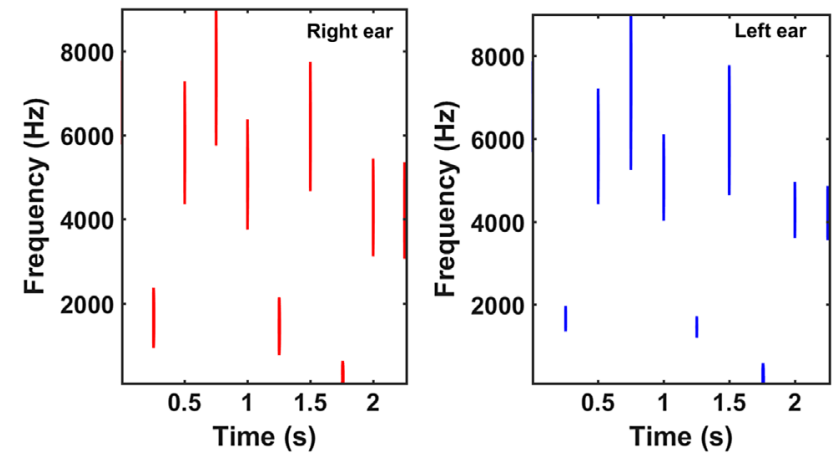

Figure 7. Spectrograms of the right and left tone-pips sequences ( $2 \mathrm{~s}, 4$ pulses/s). A $2.9 \mathrm{~ms}$ Blackman window is used with a hop of $0.22 \mathrm{~ms}$; the Fourier transform is carried out on 4096 points.

stimulus for tinnitus therapy, as it drives selectively the damaged auditory system with the corresponding auditory filter, in the way suggested by the previous works reviewed in Section 3.1.

\subsection{Matched broadband noise}

With gammatone sequences the hearing frequency band is stimulated with tones at frequencies randomly chosen and amplitudes proportional to the hearing losses at these frequencies. A broadband noise filtered by the hearing loss curve could alternatively be used. As proposed by Schaette and Kempter [45, 46] such a filtered (or coloured) broadband signal should also stimulate optimally the auditory system of the subject with amplitude proportional to the hearing loss.

A filtered broadband noise, $f b b(t)$, is then defined as,

$$
\operatorname{fbb}(t)=\mathfrak{I}^{-1}\{\operatorname{RAND}(f) \operatorname{HL}(f) A(f)\},
$$

being $\operatorname{RAND}(f)$ a random signal in the hearing frequency band, $\mathrm{HL}(f)$ is an interpolated hearing loss curve in the same frequency band, $\mathfrak{J}^{-1}$ denotes inverse Fourier transform, and $\mathrm{A}(f)$ is the constant frequency bin version of
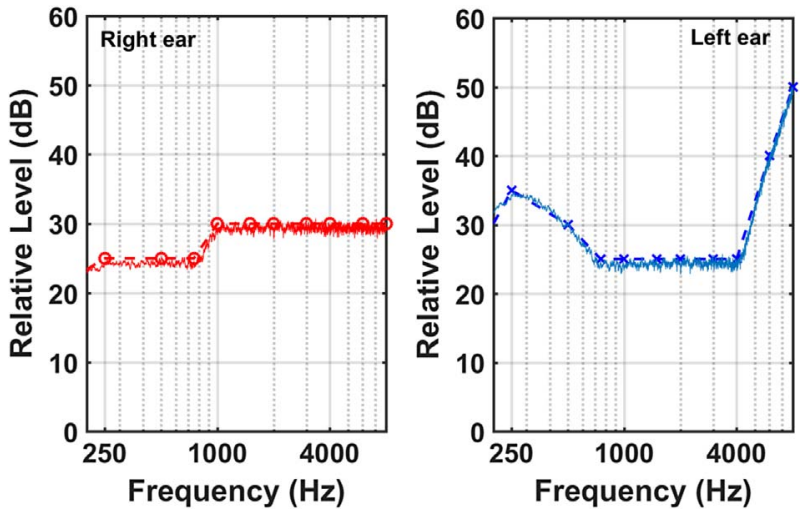

Figure 8. Log-spectra of the right and left filtered broadband stimuli superimposed to the HL curves.

the amplitude weighting function, Equation (2). The log-spectra corresponding to the right and left ears of the subject, superimposed to the hearing loss curves, are shown in Figure 8.

Although HL is considered a high risk factor to suffer of tinnitus, a percentage varying between 10 and $30 \%$ of tinnitus can occur without simultaneous hearing loss [41, 57]. Nevertheless, hearing losses above $8 \mathrm{kHz}$ [14] or cochlear synaptopathy [58], can co-occur with normal audiograms up to $8 \mathrm{kHz}$. Notice that, for these patients with flat HL, the proposed EAE with filtered broadband noise becomes a conventional TRT.

\section{Results}

The effectiveness of EAE as an optimal sound stimulus for tinnitus therapy is illustrated here with three cases extracted from our currently in process clinical study $[59,60]$. This study, approved by the CSIC Ethical Subcommittee, runs since January 2018, and is aimed to demonstrate the efficacy of short treatment (4 months, $1 \mathrm{~h}$ /day) with customised EAE in a selected cohort of Spanish tinnitus patients (150 patients until today). All the participants gave their signed informed consent to use their data for publication maintaining their anonymity.

The treatment consisted of a combination of counselling and sound therapy. Counselling was provided at the beginning of treatment, during a session of roughly $90 \mathrm{~min}$, and consisted of a presentation of the functioning of the auditory system, the mechanisms, epidemiology, and possible treatments of tinnitus with realistic expectations. Participants fulfilling the inclusion criteria were informed about the fundamentals of this therapy and requested to choose, with the help of a custom-designed graphical user interface, between either sequential (gammatones) or broadband sound, both matched to their HL curves. The principal criteria for the choice were the listening comfortability and the need to adjust the playing volume just below their tinnitus level sensation (mixing point) [23]. Most participants chose matched broadband noise, since is easier to adjust the volume to the mixing point with continuous sound. 

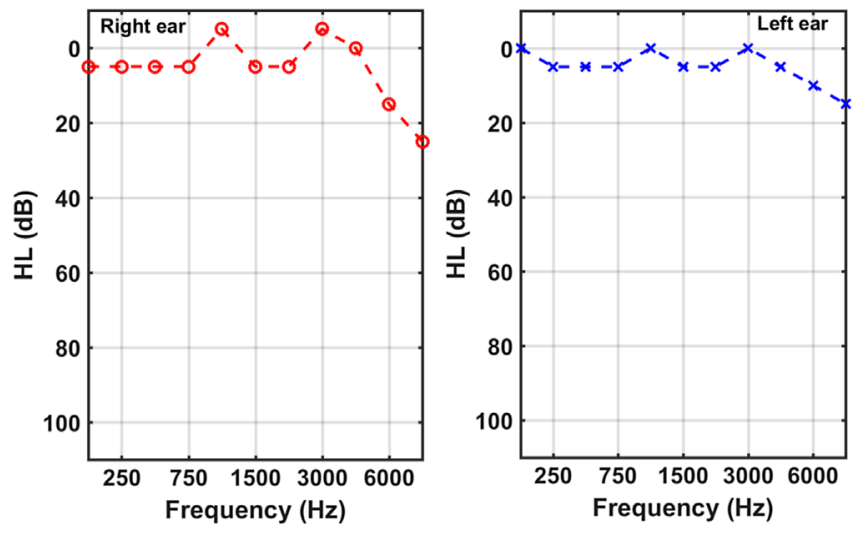

Figure 9. Right and left pure tone audiograms of a participant $\# 1$.

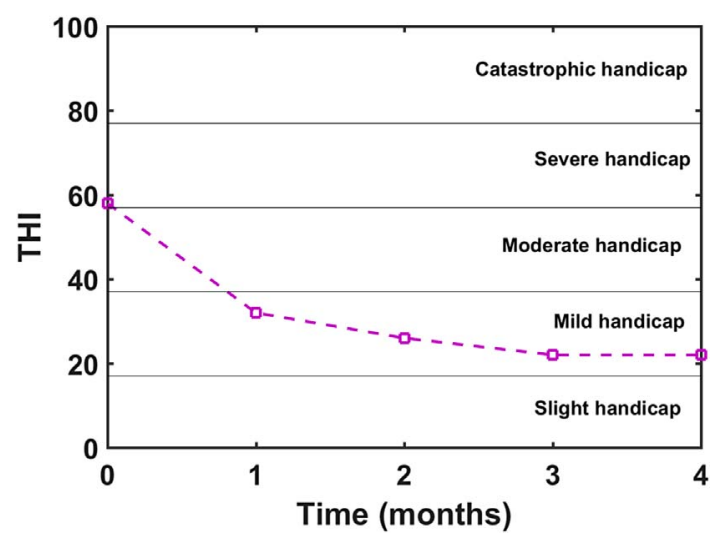

Figure 10. THI time progress of participant $\# 1$ along treatment.

The sound stimuli are designed in our laboratory, taking into account the HL curves of the patients, and are given to them in an easily playable audio format, with the prescription of listening for $1 \mathrm{~h}$ per day using high quality headphones.

Tinnitus severity of participants was assessed using a validated Spanish version of the Tinnitus Handicap Inventory (THI) [61, 62]. The handicap produced by tinnitus is graded as slight for THI $<16$, mild for $18 \leq \mathrm{THI}<36$, moderate for $38 \leq \mathrm{THI}<56$, severe for $58 \leq \mathrm{THI}<76$, and catastrophic for THI $\geq 78$ [63]. It is accepted that a reduction of THI of 20 points is clinically relevant [23].

Participant \#1 is a 39-year-old man who was included in our clinical study in December 2019. He suffered from fluctuant, severe tinnitus ( $\mathrm{THI}=58)$ at the right ear since 30 months ago, likely due to stress. His audiogram (Fig. 9) showed normal hearing except for a slight HL (25 dB HL) in the right ear at $8 \mathrm{kHz}$. His tinnitus sound was matched to a hissing of $3 \mathrm{kHz}$ with bandwidth of $15 \%$. He was treated with a matched broadband noise along four months. The time progress of his THI is illustrated in Figure 10. As it can be seen, his tinnitus handicap was reduced in two grades (from severe to mild) amounting a total THI decrease of $\Delta \mathrm{THI}=-34$.
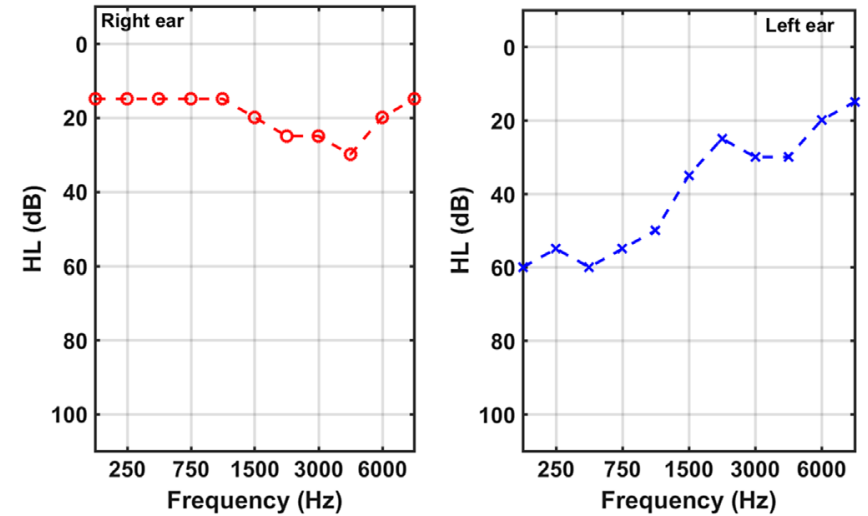

Figure 11. Right and left pure tone audiograms of a participant $\# 2$.

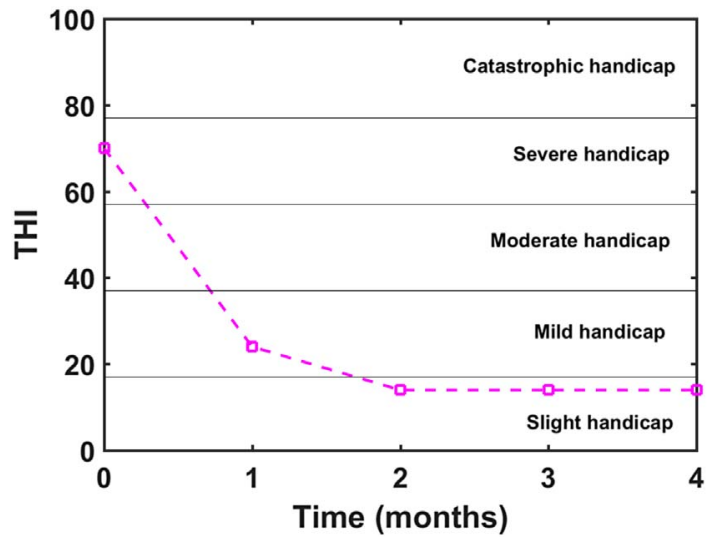

Figure 12. THI time progress of participant $\# 2$ along treatment.

Participant \#2 is a 66-year-old man who initiated our tinnitus treatment in June 2020. He was ached of fluctuant, severe tinnitus (THI $=70)$ for 6 months, likely triggered by a diagnosed ankylosing spondylitis which had caused a moderately severe conductive loss in his left ear (Fig. 11) below $1 \mathrm{kHz}$. He also displayed mild losses in both ears at middle frequencies (between 2 and $4 \mathrm{kHz}$ ). He perceived in his left ear a hissing of $300 \mathrm{~Hz}$ with bandwidth of $25 \%$. He was treated with a personalised gammatone sequence during four months. His tinnitus handicap, Figure 12, was reduced in three grades (from severe to slight) amounting a total THI decrease of $\Delta \mathrm{THI}=-56$.

Participant \#3 illustrates a mixed treatment case. She is a 45-year-old woman who enters our study in January 2018. She suffered of fluctuant, bilateral, moderate tinnitus $(\mathrm{THI}=56)$ since 14 months ago. She presented an increasing with frequency HL which varied from slight at low frequencies to moderately severe at high frequencies, Figure 13. Her tinnitus sounded as a mix of hissing at 100 and $2000 \mathrm{~Hz}$ (with bandwidth of $50 \%$ in both cases) and a kind of cymbals. She was subjected to an EAE consisting of a gammatone sequence during the first two months. After that, she changed to a matched broadband noise along the last two months. After four months of mixing 

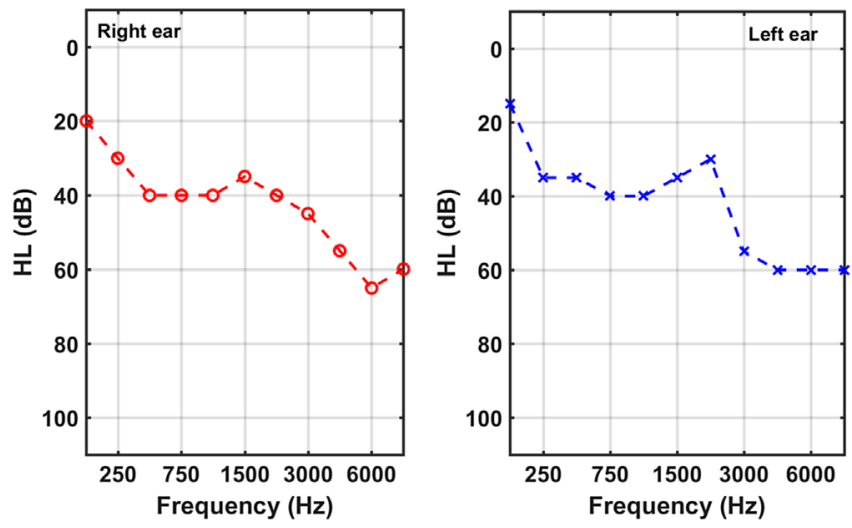

Figure 13. Right and left pure tone audiograms of a participant $\# 3$.

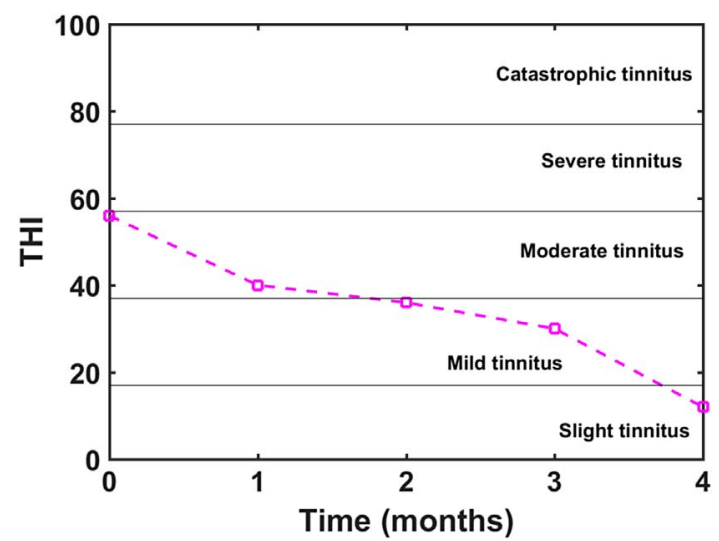

Figure 14. THI time progress of participant $\# 3$ along treatment.

EAE treatment, her THI decreased two grades (from moderate to slight), Figure 14, with a total THI reduction of $\Delta \mathrm{THI}=-44$

Therefore, independent of patient age, gender, tinnitus aetiology, tinnitus sound, or baseline handicap degree, EAE with either matched broadband noise or gammatone sequence was able to provide clinically significant tinnitus relief on these patients.

\section{Conclusions}

A sound stimulus consisting of either a sequence of gammatones or a broadband noise, both matched to the hearing loss curves of the subject, is proposed as a personalised and refined sound therapy for tinnitus. The sequential EAE already demonstrated its capability to restore the reorganized tonotopic map of cats produced by traumatizing noise, or to rescale the aberrant gain sensitivity of hyperacusis patients. The HL-matched broadband noise showed to be the optimal sound stimulation for reverse the tinnitus induced hypersensitivity in a computational model of the functioning of neurons in the auditory system. This EAE sound therapy have been applied to patients of different aetiology, age, gender or tinnitus sound, and lateralization, providing a clinically relevant handicap relief in just four months.

\section{Conflict of interest}

The authors declare that they do not have any conflict of interest.

\section{Acknowledgments}

This study was supported by CSIC grant $201750 \mathrm{E} 037$.

\section{References}

1. P.J. Jastreboff: Phantom auditory perception (tinnitus): Mechanisms of generation and perception. Neuroscience Research 8 (1990) 221-254.

2. J.J. Eggermont, P.A. Tass: Maladaptive neural synchrony in tinnitus: Origin and restoration. Frontiers in Neurology 6 (2015) 29.

3. A.F. Holm, M.J. Staal, J.J.A. Mooij, F.W.J. Albers: Neurostimulation as a new treatment for severe tinnitus: A pilot study. Otology \& Neurotology 26 (2005) 425-428.

4. J. Zhang: Auditory cortex stimulation to suppress tinnitus: Mechanisms and strategies. Hearing Research 295 (2013) 38-57.

5. F.G. Zeng, Q. Tang, A. Dimitrijevic, A. Starr, J. Larki, N.H. Blevins: Tinnitus suppression by low-rate electric stimulation and its electrophysiological mechanisms. Hearing Research 277 (2011) 61-66.

6. P. Van de Heyning, O. Meeus, C. Blaivie, K. Vermeire, A. Boudewyns, D. De Ridder: Tinnitus: A multidisciplinary clinical approach. B-ENT 3 (2007) 3-10.

7. D.A. Hall, H. Haider, D. Kikidis, M. Mielczarek, B. Mazurek, A.J. Szczepek, C.R. Cederroth: Toward a global consensus on outcome measures for clinical trials in tinnitus. Trends in Amplification 19 (2015) 1-7.

8. N. Weisz, S. Voss, P. Berg, T. Elbert: Abnormal auditory mismatch response in tinnitus sufferers with high-frequency hearing loss is associated with subjective distress level. BMC Neuroscience 5 (2004) 1-9.

9. C. Herráiz, J. Hernández: Acúfenos. Actualización. Ars Medica, Madrid, 2002. (in Spanish).

10. M.A. López, F. Esteban: Acúfeno como señal de malestar. Amplifón, Granada, 2010. (in Spanish).

11. M. Knipper, P. Van Dijk, I. Nunes, L. Rüttiger, U. Zimmermann: Advances in the neurobiology of hearing disorders: Recent developments regarding the basis of tinnitus and hyperacusis. Progress in Neurobiology 111 (2013) 17-33.

12. B. Gourevitch, J.M. Edeline, F. Occelli, J.J. Eggermont: Is the din really harmless? Long-term effects of non-traumatic noise on the adult auditory system. Nature Reviews in Neuroscience 15 (2014) 483-491.

13. M.M. Vio, R.H. Holme: Hearing loss and tinnitus: 250 million people and US $\$ 10$ billion potential market. Drugs Discovery Today 10 (2005) 1263-1265.

14. N. Weisz, T. Hartmann, K. Dohrmann, W. Schlee, A. Noreña: High-frequency tinnitus without hearing loss does not mean absence of deafferentation. Hearing Research 222 (2006) 108-114. 
15. B.T. Paul, I.C. Bruce, L.E. Roberts: Evidence that hidden hearing loss underlies amplitude modulation encoding deficits in individuals with and without tinnitus. Hearing Research 344 (2017) 170-182.

16. J.J. Eggermont: The neuroscience of tinnitus. Oxford University Press, London, 2012.

17. A.J. Noreña, J.J. Eggermont: Enriched acoustic environment alter noise trauma reduces hearing loss and prevents cortical map reorganization. The Journal of Neuroscience 25 (2005) 699-705.

18. J.P. Rauschecker, A.M. Leaver, M. Muhlau: Tuning out the noise: Limbic-auditory interactions in tinnitus. Neuron 66 (2010) 819-826.

19. M.L. Lenhardt: Tinnitus devices, in Encyclopedia of Biomaterials and Biomedical Engineering, Wnek GE, Bowling GL, Editors. CRC Press, Boca Raton, 2008, pp. 2610-2619.

20. G.D. Searchfield, M. Durai, T. Linford: A state-of-the-art review: Personalization of tinnitus sound therapy. Frontiers in Psychology 8 (2017) 1599.

21. M. Sereda, J. Xia, A. El Refaie, D.A. Hall, D.J. Hoare: Sound therapy (using amplification devices and/or sound generators) for tinnitus in adults (protocol). Cochrane Database of Systematic Reviews 8 (2018) CD013094.

22. M. Pienkowski: Rationale and efficacy of sound therapies for tinnitus and hyperacusis. Neuroscience 407 (2019) 120-134.

23. P.J. Jastreboff: 25 years of tinnitus retraining therapy. HNO 63, 3 (2015) 07-311.

24.P.J. Jastreboff, M.M. Jastreboff: Tinnitus and decreased sound tolerance, in Otorrinolaringology Head and Neck Surgery, Wackym PA, Snow JB, Editors, People's Medical Publishing House, Shelton. 2016.

25. H. Flor, D. Hoffmann, M. Struve, E. Diesch: Auditory discrimination training for the treatment of tinnitus. Applied Psychophysiology and Biofeedback 29 (2004) 113-120.

26. C. Herráiz, I. Diges, P. Cobo, G. Plaza, J.M. Aparicio: Auditory discrimination therapy (ADT) for the tinnitus management: preliminary results. Acta Oto-Laryngologica 126 (2006) 80-83.

27. C. Herráiz, I. Diges, P. Cobo: Auditory discrmination therapy (ADT) for tinnitus management. Progress in Brain Research 166 (2007) 467-471.

28. C. Herráiz, I. Diges, P. Cobo, J.M. Aparicio: Cortical reorganization and tinnitus: principles of Auditory Discrmination Therapy (ADT) for tinnitus management. European Archives of Oto-Rhino-Laryngology 266 (2009) 9-16.

29. C. Herráiz, I. Diges, P. Cobo, J.M. Aparicio, A. Toledano: Auditory discrimination training for tinnitus treatment: The effect of different paradigms. European Archives of Oto-Rhino-Laryngology 267 (2010) 1067-1074.

30. R.I. Lipman, S.P. Lipman: Phase-shift treatment for predominant tone tinnitus. Otolaryngology-Head and Neck Surgery 136 (2007) 763-768.

31. K. Vermeire, K. Heyndrickx, D. De Ridder, P. Van de Heyning: Phase shift tinnitus treatment: An open prospective clinical trial. B-ENT Supp. 7 (2007) 65-69.

32. P.B. Davis, B. Paki, P.J. Hanley: Neuromonics tinnitus treatment: Third clinical trial. Ear \& Hearing 28 (2007) 242-259.

33. H. Okamoto, H. Stracke, W. Stoll, C. Pantev: Listening to tailor-made notched music reduces tinnitus loudness and tinnitus-related auditory cortex activity. Proceedings of the National Academy of Sciences of the United States of America 107 (2010) 1207-1210.

34. H. Stracke, H. Okamoto, C. Pantev: Customized notched music training reduces tinnitus loudness. Communicative \& Integrative Biology 3 (2010) 1-4.
35. C. Pantev, H. Okamoto, H. Teinmann: Music-induced cortical plasticity and lateral inhibition in the human auditory cortex as foundations for tonal tinnitus treatment. Frontiers in Systems Neuroscience 6 (2012) 50.

36. I. Adamchic, C. Hauptmann, P.A. Tass: Changes of oscillatory activity in pitch processing network and related tinnitus relief induced by acoustic CR neuromodulation. Frontiers in Systems Neuroscience 6 (2012) 18.

37.P.A. Tass, O.V. Popovych: Unlearning tinnitus-related cerebral synchrony with acoustic coordinated reset stimulation: Theoretical concept and modelling. Biological Cybernetics 106 (2012) 27-36.

38. W. Wurzer, C. Hauptmann: Clinical experiences with acoustic CR neuromodulation in patients with chronic tinnitus. Journal of Clinical Studies \& Medical Case Reports 5 (2018) 059.

39. R.W. Sweetow, J.H. Sabes: Effects of acoustical stimuli delivered through hearing aids on tinnitus. Journal of the American Academy of Audiology 21 (2010) 461-473.

40. M. Herzfeld, E. Ciurlia-Guy, R.W. Sweetow: Clinical trial on the effectiveness of Widex Zen Therapy for tinnitus. Hearing Review 21 (2014) Available at: http://www.hearingreview. com/2014/10/clinical-trial-effectiveness-widex-zen-therapytinnitus.

41. D.J. McFerran, D. Stckdale, R. Holme, C.H. Large, D.M. Baguley Why is there no cure for tinnitus? Frontiers in Neuroscience 13 (2019) 802.

42. K.S. Kraus, B. Canlon: Neuronal connectivity and interactions between the auditory and limbic systems. Effects of noise and tinnitus. Hearing Research 288 (2012) 34-46.

43. A.M. Leaver, L. Renier, M.A. Chevillet, S. Morgan, H.J. Kim, J.P. Rauschecker: Dysregulation of limbic and auditory networks in tinnitus. Neuron 69 (2011) 33-43.

44. J.A. Henry, M.A. Schechter, T.L. Zaugg, S. Griest, P.J. Jastreboff, J.A. Vernon, C. Kaelin, M.B. Meikle, K.S. Lyons, B.J. Stewart: Outcomes of clinical trial: Tinnitus masking versus tinnitus retraining therapy. Journal of the American Academy of Audiology 17 (2006) 104-132.

45. R. Schaette, R. Kempter: Development of tinnitus-related neuronal hyperactivity through homeostatic plasticity after hearing loss: A computational model. European Journal of Neuroscience 23 (2006) 3124-3138.

46. R. Schaette, R. Kempter: Development of hyperactivity after hearing loss in a computational model of the dorsal cochlear nucleus depends on neuron response type. Hearing Research 240 (2008) 57-72.

47. A.J. Noreña, S. Chery-Croze: Enriched acoustic environment rescales auditory sensitivity. NeuroReport 18 (2007) 12511255 .

48. C. Herráiz, I. Diges, P. Cobo, A.J. Noreña, J. Hernández, J. M. Aparicio: Enriched acoustic environment with spectrum matched to the patient hearing loss curve for tinnitus sound therapy, in International Tinnitus Seminar, Florianopolis (Brasil). 2011.

49. D.J. Hermes, J.J. Eggermont, A.M.H.J. Aertsen, P.I.M. Johannesma: Spectro-temporal characteristics of single units in the auditory midbrain of slightly anaesthetised grass fogs investigated with tone stimuli. Hearing Research 6 (1983) $103-126$.

50. A.J. Noreña, J.J. Eggermont: Changes in spontaneous neural activity immediately after an acoustic trauma: Implication for neural correlates of tinnitus. Hearing Research 183 (2003) 137-153.

51. T.S. Korn: Theory of audio information. Acustica 22 (1969/ 70) $336-344$. 
52. M. Slaney: An efficient implementation of the PattersonHoldsworth auditory filter bank. Apple Computer Technical Report \#35, Apple Computer Inc., 1993.

53. T. Irino, R.D. Patterson: A time-domain, level-independent auditory filter: The gammachirp. Journal of the Acoustical Society of America 101 (1997) 412-419.

54. R.F. Lyon, All-pole models of auditory filtering, in Diversity in Auditory Mechanics, E.R. Lewis, G.R. Long, R.F. Lyon, P.M. Narins, C.R. Steele, E. Hecht-Poinar, Editors, World Scientific Publishing, Singapore. 1997.

55. A.G. Katsiamis, E.M. Drakakis, R.F. Lyon: Practical gammatone-like filters for auditory processing. EURASIP Journal of Audio Speech and Music Processing (2007). 63685.

56. R.D. Patterson: The sound of a sinusoid: Spectral models. Journal of the Acoustical Society of America 96 (1994) 14091418.

57. A.C. Lindblad, U. Rosenhall, A. Oloffson, B. Hagerman: Tinnitus and other auditory problems - Occupational noise exposure below risk limits may cause inner ear dysfunction. PLoS One 9 (2014) e97377.

58. M.C. Liberman, S.G. Kujawa: Cochlear synaptopathy in acquired sensorineural hearing loss: Manifestations and mechanisms. Hearing Research 349 (2017) 138-147.
59. M. Cuesta, P. Cobo: Broadband sound equalized by the hearing loss curves as an improved stimulus for tinnitus retraining therapy - A pilot, non-controlled observational study. The Journal of International Advanced Otology 16, 2 (2020) 207-212.

60. P. Cobo: Successful application of sound therapy to a patient with severe tinnitus: Case study. Journal of Otolaryngology, Head \& Neck Surgery 7 (2021) 051.

61. C.W. Newman, S.A. Sandridge, G.P. Jacobson: Psychometric adequacy of the tinnitus handicap inventory (THI) for evaluating treatment outcome. Journal of the American Academy of Audiology 9 (1998) 153-160.

62. C. Herráiz, F.J. Hernández Calvín, G. Plaza, M.C. Tapia, G. de los Santos: Evaluación de la incapacidad en los pacientes con acufenos [Handicap assessment in tinnitus patients]. Acta Otorrinolaringológica Española 52 (2001) 534-538. (in Spanish).

63. A. McCombe, D. Baguley, R. Coles, L. McKenna, C. McKinney, P. Windle-Taylor: Guidelines for the grading of tinnitus severity: The results of a working group commissioned by the British Association of Otolaryngologists, Head and Neck Surgeons. Clinical Otolaryngology \& Allied Sciences 26, 2001 (1999) 388-393.

Cite this article as: Cobo P, Cuesta M \& de la Colina C. 2021. Customised enriched acoustic environment for sound therapy of tinnitus. Acta Acustica, 5, 34 . 